\title{
Role of 18F-FDG PET/CT in the diagnosis of inflammatory and infectious
} vascular disease

\author{
Beata Chrapko', Marek Chrapko², Anna Nocuń, Bogusław Stefaniak¹, Tomasz Zubilewicz², Andrzej Drop ${ }^{3}$ \\ ${ }^{1}$ Chair and Department of Nuclear Medicine, Medical University of Lublin \\ ${ }^{2}$ Chair and Clinic of Vascular Surgery and Angiology, Medical University of Lublin \\ ${ }^{3}$ Department of Radiology, Medical University of Lublin
}

[Received 8 VI 2015; Accepted 8 IX 2015]

\begin{abstract}
This review article discusses the utility of 18F-FDG PET/CT in diagnosis and management of vascular disease. We stress usefulness of this method in large vessel inflammation and infection.

In our work we based on the literature analysis and clinical cases diagnosed in our institution by use of 18F-FDG PET/CT. The literature exploration was focusing on vascular inflammation and infections and 18-FDG PET. The search was performed on PubMed database and cross referencing.

We present the practical review with several images of vascular diseases like: Takayasu arteritis, giant cell arteritis, vascular graft infections, abdominal aortic aneurysm infections and cases of aortitis and periaortitis. From this work inflammation associated with atheromatic process and vulnerable atherosclerotic plaque we excluded.

18F-FGD PET/CT is a sensitive metabolic, reliable, non-invasive imaging modality suitable for diagnosis and follow-up of inflammation and infections in vascular system.
\end{abstract}

KEY words: positron emission tomography, fluoro-deoxy-glucose, large vessel vasculitis, vascular graft infections, aortitis, periaortitis, abdominal aortic aneurysm infections

Nuclear Med Rev 2016; 19, 1: 28-36

\section{Background}

Increase application of positron emission tomography/computed tomography (PET/CT) with use of 18F-fluoro-2-deoxy-D-glucose (18F-FDG) in the diagnosis of inflammatory and infectious diseases is recently observed. As a result of this interest, combine guidelines of the European Association of Nuclear Medicine (EANM) and the Society of Nuclear Medicine and Molecular Imaging (SNMMI) were published in 2013 [1]

18F-FDG primarily was proposed in oncologic PET/CT. $18 \mathrm{~F}-\mathrm{FGD}$ as glucose analogue is taken up by viable cells using membrane glucose transporter proteins (GLUT) and subsequently is phosphorylated by hexokinase. During routine imaging of cancer patients, accumulation of 18F-FDG was observed at sites of inflammation/infection. Inflammatory cells involved in that reaction — like monocytes and neutrophils — are also able to overexpress GLUT 1 and GLUT 3 and have high activity of hexokinase. This ability predominantly depends on glycolytic activity of these cells and is based

Correspondence to: Beata E. Chrapko, MD

Chair and Department of Nuclear Medicine, Medical University of Lublin

Jaczewskiego 8c, 20-954 Lublin, Poland

Tel./fax: + 48817244339

E-mail: beata.chrapko@wp.pl on the phenomenon that granulocytes, essentially neutrophils and monocytes, use glucose as an energy source only after activation during the metabolic burst $[1,2]$. Thus, the accumulation of 18F-FDG in activated inflammatory cells enables the imaging of inflammation and infections. Hence, 18F-FDG PET/CT is designated for diagnosis of various inflammatory and infectious diseases, such as: sarcoidosis, inflammatory bowel disease, osteomyelitis, spondylodiscitis, fever of unknown origin (FUO), primary evaluation of vasculitides, vascular prostheses infection, and infection of intravascular devices, pacemakers and catheters [1]. Additionally, the hybrid approach of FDG PET/CT gives an complementary information about inflammatory or infectious - increased FDG uptake is seen mainly in active processes, whereas CT provides information of the advance stages, for example calcification in chronic or past inflammation [3]. To avoid false positive results in vascular diseases evaluation, one should be aware that in physiological conditions, large arteries like: aorta, iliac and femoral, subclavian and carotid arteries, present homogeneously increased activity of FDG. Arterial wall activity increases with age and also in patients with hypertension and hyperlipidemia. Increased arteries activity of FDG has been reported in $31-59 \%$ of cancer patients over 50 years of age [3]

This review is based on the literature analysis and clinical cases diagnosed at our institution by use of $18 \mathrm{~F}-\mathrm{FDG}$ PET/CT. The literature exploration was focused on vascular inflammation and 
Table 1. Classification of non-infectious vasculitis

\begin{tabular}{|ll}
\hline Size of vessels & Type of vasculitis \\
\hline Large vessels & Takayasu's arteritis \\
& Giant cell arteritis \\
Medium vessels & Periarteritis nodosa \\
& Kawasaki's arteritis \\
& Primary CNS vasculitis \\
& Thrombangitis obliterans (Buerger's disease) \\
& Wegener's disease \\
Small vessels & Churg-Strauss syndrome \\
& Microscopic polyangiitis \\
& Henoch-Schonlein purpura \\
& Essential cryoglobulinemia vasculitis \\
& Cutaneous leucocytoplastic angiitis
\end{tabular}

infections and 18-FDG PET. The literature search was performed on PubMed database by use of the combination of "FDG", "vasculitis", "vascular infection", "vascular graft infection" and "aortitis". The manual cross referencing were also done.

This review pays special attention to the utility of FDG PET/CT in diagnosis and management of inflammatory and infections vascular diseases. From this work we excluded inflammation associated with atheromatic process and vulnerable atherosclerotic plaque.

All images presented in this paper were obtained in Chair and Department of Nuclear Medicine Medical University of Lublin. They were prepared using PET/CT system Biograph mCT S(64)-4R (Siemens, Germany). The majority of patients were sent from Chair and Clinic of Vascular Surgery and Angiology Medical University of Lublin, after giving written consent.

\section{Vasculitis}

Vasculitis is an inflammation of vessel walls with leukocyte infiltration and reactive destruction of mural structures and adjacent tissue. The inflammation site avidly accumulates glucose and 18 F-FDG.

As far as etiology is concerned, vasculitis could be classified either as infectious or noninfectious. Most forms of noninfectious vasculitis are divided according to the vessel size involved. This classification, as in Chapel-Hill consensus conference, is presented in Table 1. The infectious vasculitis can be bacterial (e.g., Salmonella or Stphylococcus), luetic (syphilis), mycobacterial (e.g., Mycobarcterium tuberculosis), viral (e.g., HIV), rickettsial as well as fungal (e.g., Aspergillus arteritis) [4-6].

Although there are sporadic reports on the use of $18 \mathrm{~F}-\mathrm{FDG}$ PET in a variety of vasculitides of small and medium size vessels such as polyarteritis nodosa, Churg-Strauss syndrome or Wegener's granulomatosis, this technique is used at present mainly in large vessel vasculitis $[3,7]$. PET technique due to limited spatial resolution is not yet a reliable tool for the assessment of the medium and small size-vessel vasculitis. The large vessel vasculitides like: Takayasu arteritis, giant cell arteritis and other cases of aortitis and periaortitis are of interest with respect to 18F-FDG PET/CT.

Takayasu arteritis (TA), known also as pulseless disease, is sort of rare vascular disease, affecting 2-3 patients per year per million in worldwide population, with predilection to young Asian female aged 15-20 years. This disease is ten times more frequent among females than males [8]. This necrotizing and obliterative large-vessel

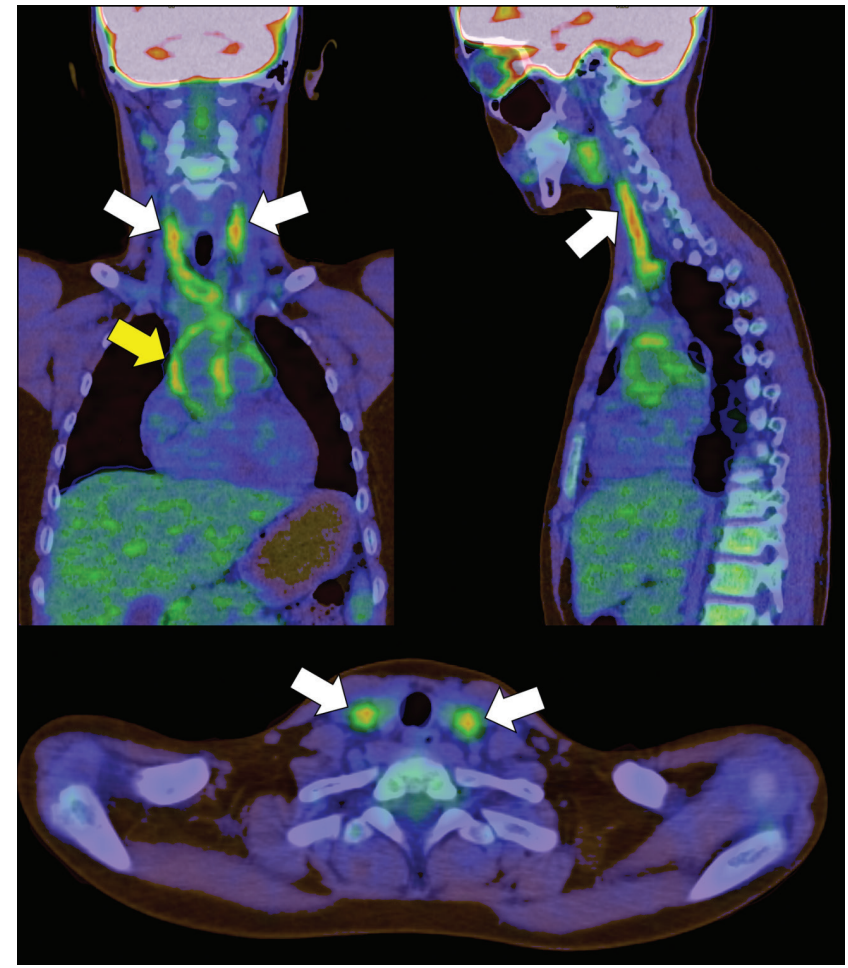

Figure 1. Takayasu arteritis in a 14-year-old boy who was referred due to fever of unknown origin. He presented weight loss, weakness and anemia. The symptoms occurred two months before the examination. 18F-FDG PET/CT findings: high uptake of FDG in the ascending aorta (yellow arrow) and in both common carotid arteries (white arrows)

arteritis is usually treated by a high dose of glucocorticoids. The early stage of the disease is manifested by systemic symptoms, such as fever, malaise, night sweats, weakness and pain. In this stage granulomas and infiltration by mononuclear cells occur in the adventitia and media. Manifestation of the late stage, known as occlusive or pulseless phase, depends on the territory of arterial occlusion. Commonly, the disease involves abdominal aorta, than descending aorta, aortic arch and branch vessels. In the late stage calcification and stenosis lead to narrowing and occlusions of the involved arteries. Less frequently, inflammatory destruction of media, leads to aneurysmal dilatation of arteries.

The diagnosis of TA is based on clinical symptoms which depend on disease activity and the degree of stenosis of involved arteries. Markers of inflammation, such as serum C-reactive protein (CRP) and erythrocyte sedimentation rate (ESR) levels as well as 18F-FDG PET/CT help to assess the disease activity. Figure 1 shows the case of young boy, who presented with unspecific symptoms like fever, malaise, weight lost, weakness, anemia. He was sent to our institution as FUO, but he was actually in the acute phase of TA. His carotid arteries were also involved and hypometabolic regions in the brain were seen.

18F-FDG PET/CT can be helpful in assessment of disease activity before treatment as well as to monitor therapeutic effect or to detect relapse of the disease despite the therapy [9]. An example of active phase of TA before treatment and 6 months after glucocorticoids treatment with some normalization of wall vessel activity is shown in Figure 2. In the active phase of TA high uptake of 18F-FDG in PET/CT study is observed, whereas in inactive disease 


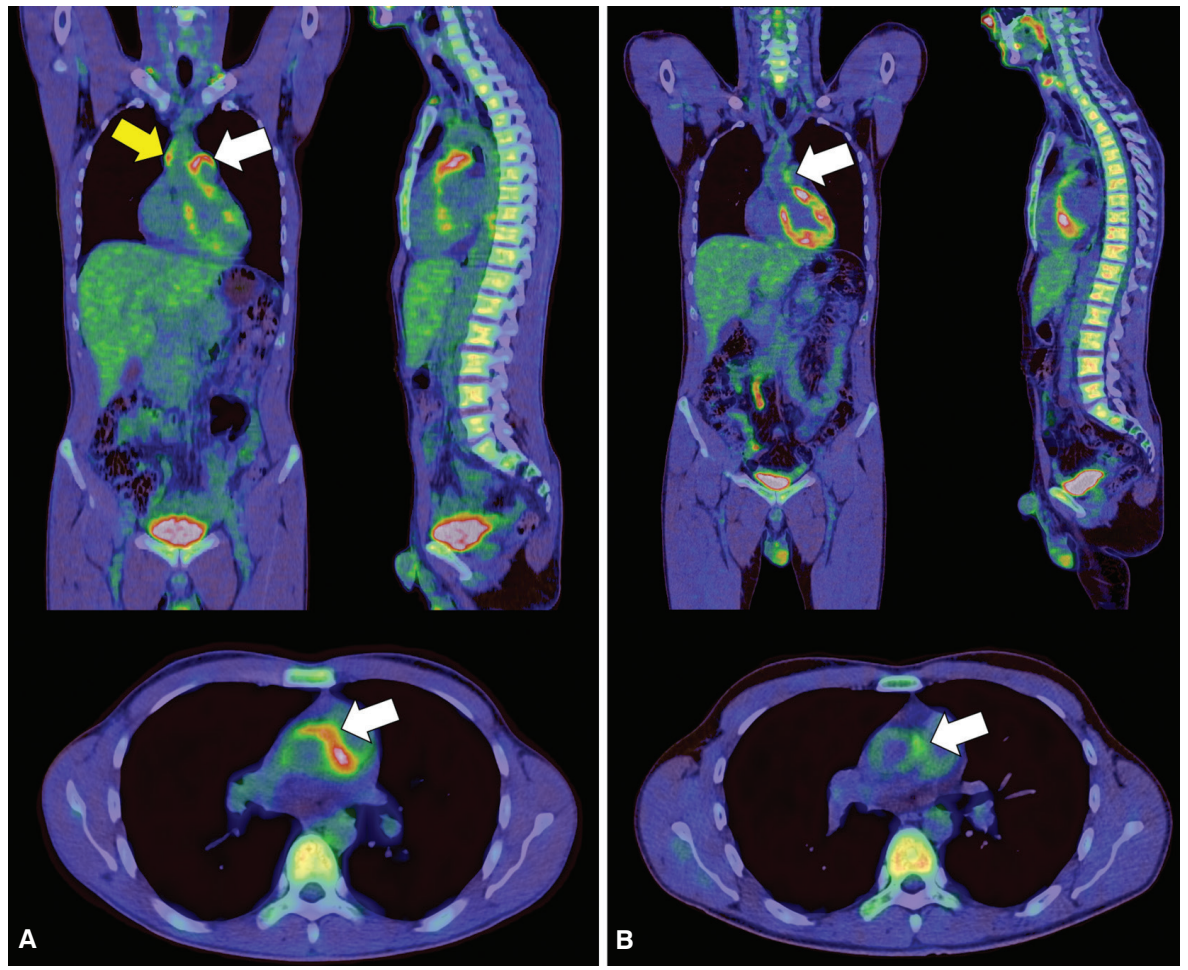

Figure 2. Takayasu arteritis located mainly in the pulmonary trunk in a 33-year-old male patient. Diagnosis was based on an echocardiography and confirmed in contrast enhanced CT. A. 18F-FDG PET/CT examination before treatment. High accumulation of FDG in the pulmonary trunk (white arrow) and in the ascending aorta (yellow arrow); B. 18F-FDG PET/CT examination 6 months after treatment, some normalization of vascular metabolism in the pulmonary trunk (white arrow) and in the ascending aorta (yellow arrow) is seen (not complete remission).

this uptake is decreased. The estimation of biodistribution of FDG is based on visual assessment or/and semiquantitative analysis, for example by evaluation of maximum standardized uptake value (SUV max).

Tezuka et al. proposed the cutoff values for active TA SUV $\max \geq 2.1, \mathrm{CRP}>0.2 \mathrm{mg} / \mathrm{dl}$ and $\mathrm{ESR} \geq 19 \mathrm{~mm} / \mathrm{h}$ [9]. They observed rising of SUV max in active phase of disease: in untreated patients - to SUV max 2.8 (2.4-3.2) and in patients with relapse of treatment - SUV max 2.6 (2.3-3.3). They also recommend FDG PET to monitor the effects of the treatment. Different cutoff SUV values were proposed by Kobayashi et al., where SUV $>1.3$ indicates active vasculitis in TA [10].

Aortic wall thickening in contrast enhanced $\mathrm{CT}$, described in the early stage of TA, is typically presented as so-called "double ring" [11]. In chronic cases of TA, arterial calcification is observed after 5 years or more. In magnetic resonance (MR) imaging early wall thickening can be depicted as well as mural edema. Both CT and MR show stenosis, mural thrombi, thickening of aortic valves cusp and peripheral effusion [12-14].

In contrast to TA, giant cell arteritis (GCA) is common in Caucasian population, with incidence of 18 per 100,000 habitants. Women over 50 years of age (female:male; $2: 1$ ) are particularly affected [15]. Etiology of GCA remains unknown. The most typical symptoms are: headache in two thirds of patients and polymyalgia rheumatica in half of cases. Myalgia, neck pain, scalp tenderness, jaw claudication, fever, transient ischemic attack, fatigue, anorexia, weight lost, and night sweats are also frequent [16]. GCA mainly involves the complex of intima-media. The acute stage is characterized by granulomatous infiltration, mainly by giant cells and lymphocytes [7]. In chronic stage, progressive fibrosis of arterial wall occurs. This arteritis affects usually cranial artery, in particular superficial temporal artery, but can involve entire aorta and its branches as well as coronary, mesenteric and vertebral arteries. High uptake of FDG in wall of affected arteries is usually present (Figure 3 ).

The exacerbation of disease is associated with significantly reduced 5-year survival [17]. The aortic involvement happens in 15\% of GCA cases. Moreover, as reported Pacini et al. [18], GCA is the most common aortitis and represents $75 \%$ of cases of this disease in North America. The large vessel vasculitis, particularly GCA, represents up to $17 \%$ of fever of unknown origin [19].

The diagnosis of GCA is usually based on biopsy of temporal artery; however, in case of aortitis, it is not appropriate because of the frequent inflammation of the extracranial arteries without involvement of the temporal arteries [20].

Among various diagnostic modalities, increased role of FDG PET has been recently underlined in detection and monitoring of GCA. FDG PET has been shown to be sensitive for extracranial artery involvement with sensitivity $56 \%$, specificity $98 \%$, positive predictive value $93 \%$ and negative predictive value $80 \%$ [21]. In the semiquantitative analysis of aortitis in $\mathrm{PET} / \mathrm{CT}$, where images were obtained 180 minutes after 18F-FDG administration, the target (aortic wall) to background (aortic lumen activity) ratio (TBR) was defined. The TBR index of 1.34 shows very high accuracy (sensitivity $100 \%$ and specificity $94.4 \%$ ) and is strongly recommended for the diagnosis of aortitis in the clinical settings [20].

The limitation of FDG PET in assessment of cranial artery inflammation is low spatial resolution and, therefore, it cannot replace the biopsy of the temporal artery [22]. Another limitation of FDG PET 


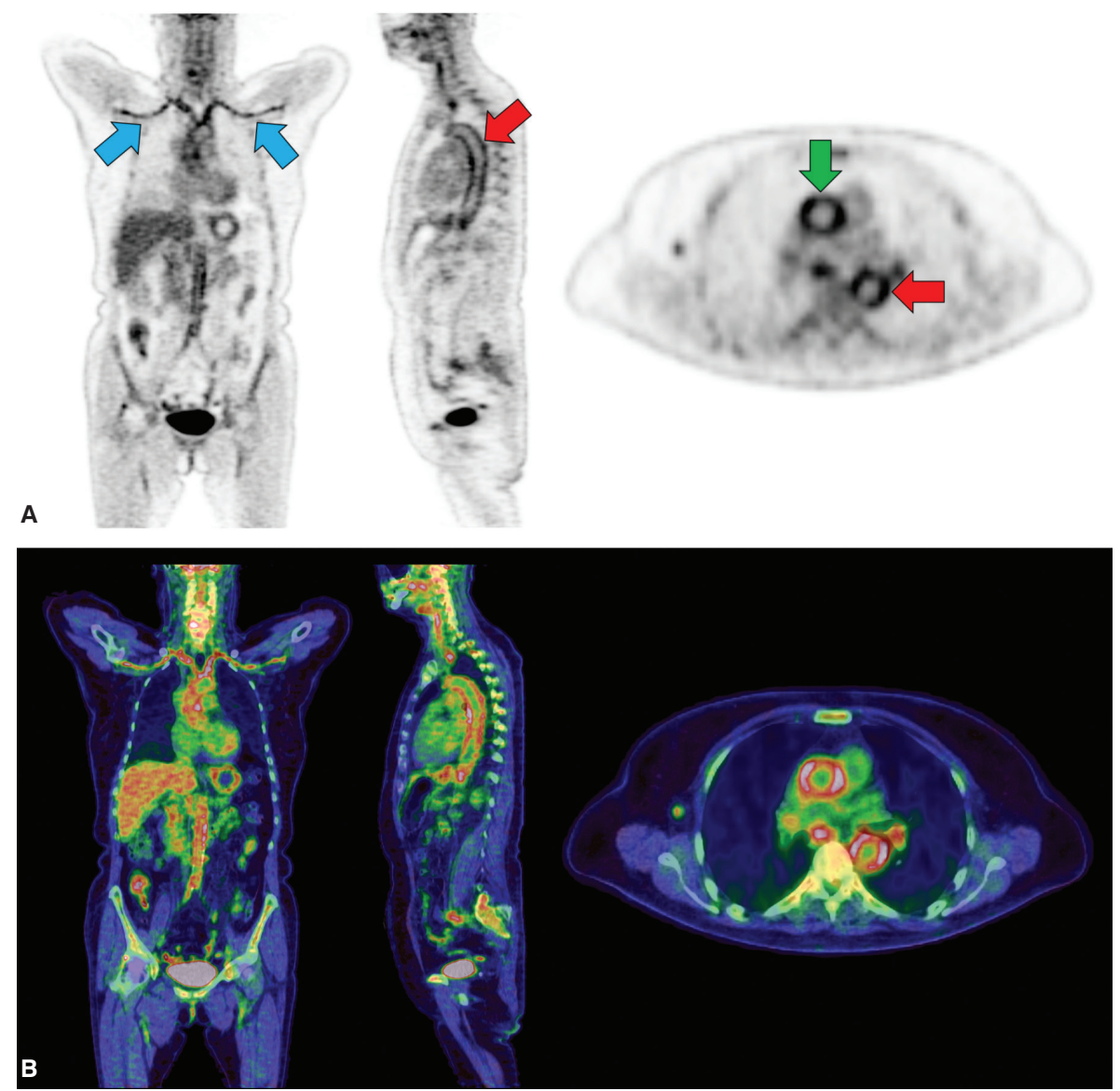

Figure 3. 18F-FDG PET/CT of a 65-year-old female patient, referred by oncologist with unspecific symptoms and suspicion of lymphoma. 18F-FDG PET/CT examination detected high metabolism in large vessels. The final diagnosis — giant cell arthritis. A. High uptake of FDG in the subclavian arteries (blue arrows on coronal view), aorta (red arrow on sagittal view), and in the ascending and descending aorta respectively (red and green arrows, on axial slice); B. Fused image of 18 F-FDG PET/CT coronal, sagittal and axial views

is that vessel activity and SUV should be interpreted with caution because of normal blood pool activity and atheromatous changes in the vessel wall. It has to be also taken in account that vascular uptake of FDG increases with age, and in the age of 60 the mean SUV can be up to $2.01 \pm 0.5$ [23]. As Bural et al. conclude, "Increased FDG uptake likely represents the presence of active inflammatory process of atherosclerotic plaque. The magnitude of inflammation within the wall of the large arteries increases with aging" [23].

\section{Inflammation of aortic abdominal aneurysms}

By definition, aortic abdominal aneurysm (AAA) is diagnosed when infra-renal aortic diameter exceeds $3 \mathrm{~cm}$ [24]. Major risk factors of AAA include age ( $\geq 65$ years of age), male gender and a history of ever smoking ( $\geq 100$ in person's lifetime) [25]. Most dangerous complication of AAA is rupture which is fatal in $70-90 \%$ of cases and is the $13^{\text {th }}$ cause of death in Western countries [26]. Therefore, prediction of AAA rupture is essential. According to several authors [27-29], the strongest risk factor of AAA rupture is maximal aortic diameter. Nevertheless, in clinical practice ruptures of small AAA also occur and its incidence ranges from 2 to $8 \%[30,31]$. The main role in the small AAA rupture is played by active inflammatory processes, which can be visualized by 18F-FDG PET/CT [32]. Figure 4 presents active phase of inflammation of AAA. The inflammation predominantly affects the adventitia and consists of dense infiltration of proliferating lymphocytes and phagocytes [33, 34]. Matrix metalloproteinases (MMPs) are mainly involved in elastin and collagen degradation [35]. The process preceding rupture is connected with high $\mathrm{C}$-reactive protein plasma level, loss of smooth muscle cells in the media, collagen and elastin degradation, which leads to instability and weakening of aneurysmal wall [32]. High uptake of FDG in AAA is associated with clinical symptoms, mostly with abdominal pain [36].

Recently in the multicenter study by Barwick et al., large groups with aneurysms $(n=150)$ and matching non-aneurysm controls $(n=159)$ were compared. It was found that there was no correlation between the size of AAA and the level of activity in the aortic wall, as assess by 18F-FDG PET/CT [37].

\section{Vascular graft infection}

Vascular and endovascular surgeries are usually performed in highly aseptic conditions, so infection and inflammation in these procedures are rare and amount to $0.5-5 \%$ of implantations [38]. The lowest incidence of inflammation is observed in intra-abdominal prostheses, whereas the highest one - in axillo-femoral prostheses [39]. Vascular graft infection (VGI), however rare, brings very severe complications with high morbidity and mortality, ranging between $20 \%$ and $75 \%$ [40, 41]. Factors predisposing to VGI are: prolonged preoperative stay, postoperative bacteremia, end-stage 


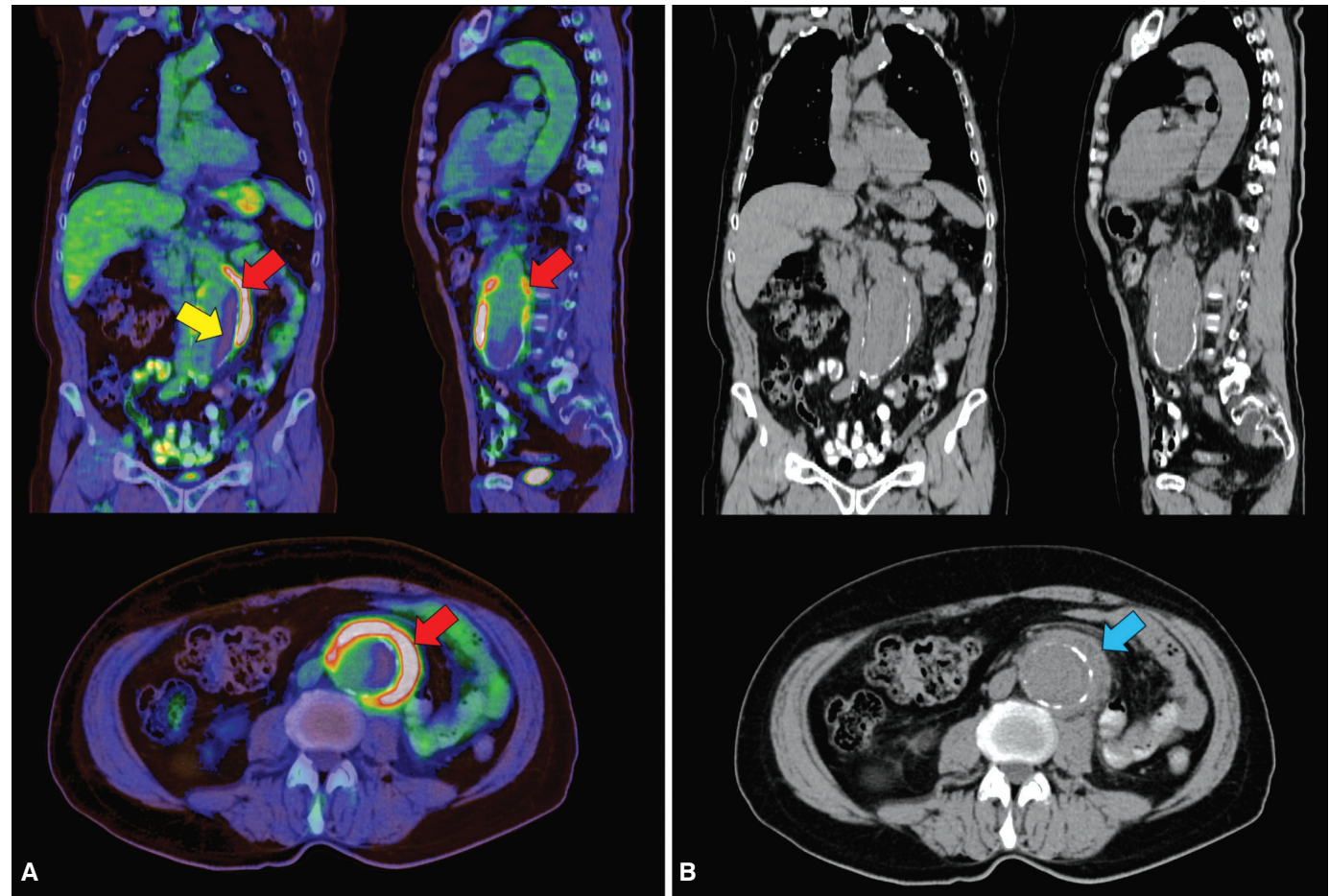

Figure 4. Inflammation of the aortic abdominal aneurysm in a 65-year-old male patient. A. Fused 18FDG PET/CT, note high uptake of FDG (red arrow) in the aneurysm wall with small area of low uptake in left-lateral aspects of the aorta (parietal thrombus, yellow arrow); B. CT without contrast media of the same patient with fluid around the aortic abdominal aneurysms (blue arrow)

renal disease, obesity, malnutrition/older age, low serum albumin, smoking, diabetes mellitus, prior irradiation, autoimmune disease/corticosteroid therapy, malignancy and chemotherapy [39]. The clinical manifestation of VGI may occur long after surgery; often may be unspecific and subtle.

Because of high-risk associated with reoperation, it is necessary to obtain information concerning presence and extend of infection. The early and reliable diagnosis is essential. Commonly, in daily clinical practice the diagnosis of VGl is based on contrast enhanced CT or MR, blood cultures and biochemical analysis. Sometimes, however, these procedures are inconclusive, especially in low-grade infections where a sensitivity and specificity of CT are about $55 \%$ and $100 \%$ [42], so more physiological approach is needed [43]. As in other inflammatory cases, increased FDG uptake is seen in VGI in areas of activated granulocytes [44]. Typically, in infected grafts focal and high uptake of FDG is observed over the prosthesis (Figure 5), whereas mild or sometimes quite high but linear activity along the grafts is rather related with inflammation after surgery or reaction of the body to foreign material (Figure 6). The appearance of the latter is related with false positive results [44].

The most difficult in the diagnosis and with highest mortality and morbidity rate are the complications associated with vascular prostheses in the chest. Tokuda et al. proposed the cut-off value of FDG uptake in thoracic aorta prosthesis (SUV max > 8) providing maximal sensitivity and specificity for differentiation between infected and non-infected graft (sensitivity: 1.0 and specificity: 0.8) [43]. Figure 7 presents examples of thoracic aorta prosthesis complicated by infections one month after implantation. For evaluation of VGI, most authors compare FDG uptake in prosthesis with surrounding tissue [45-47]. Inter alia, Fukuchi et al. applied

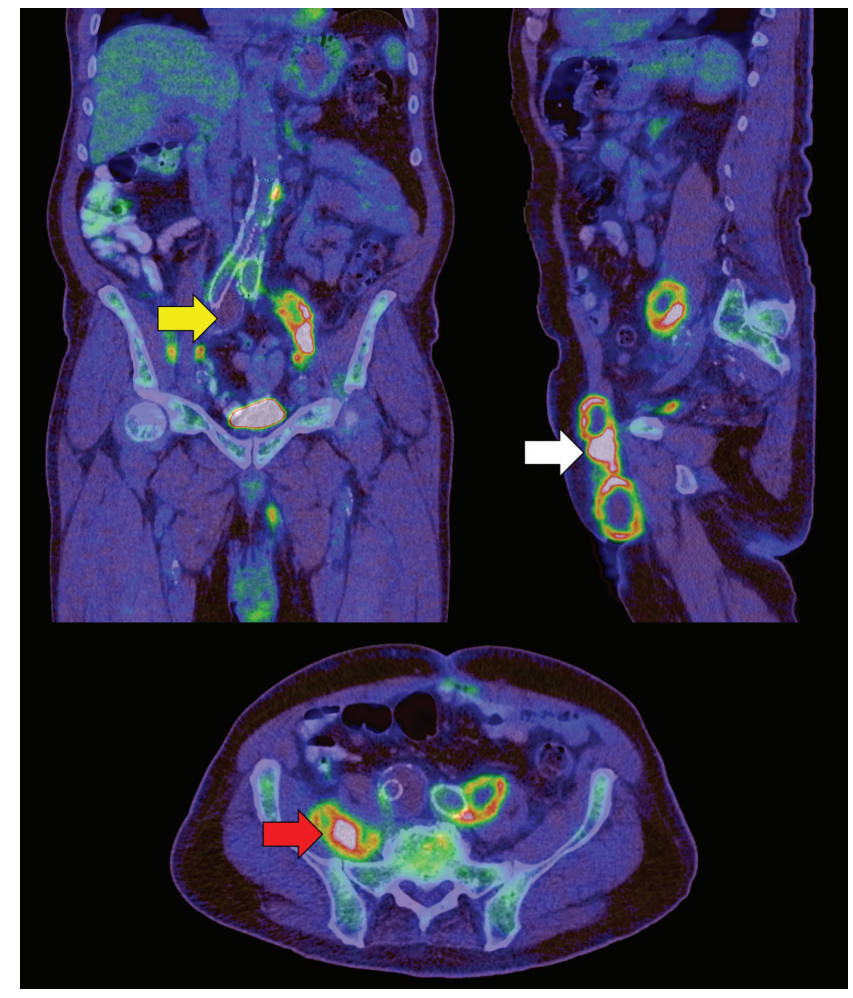

Figure 5. Infection of aorto-bi-iliac stent graft in a 76-year-old male patients, 3 years after implantation. Infection along the prosthesis, multiple abscesses in the abdomen, pelvis, groin and scrotum (white arrow). Active infection infiltrating the right psoas major muscle (red arrow). Aneurysm of the right common iliac artery with parietal thrombus (yellow arrow) 

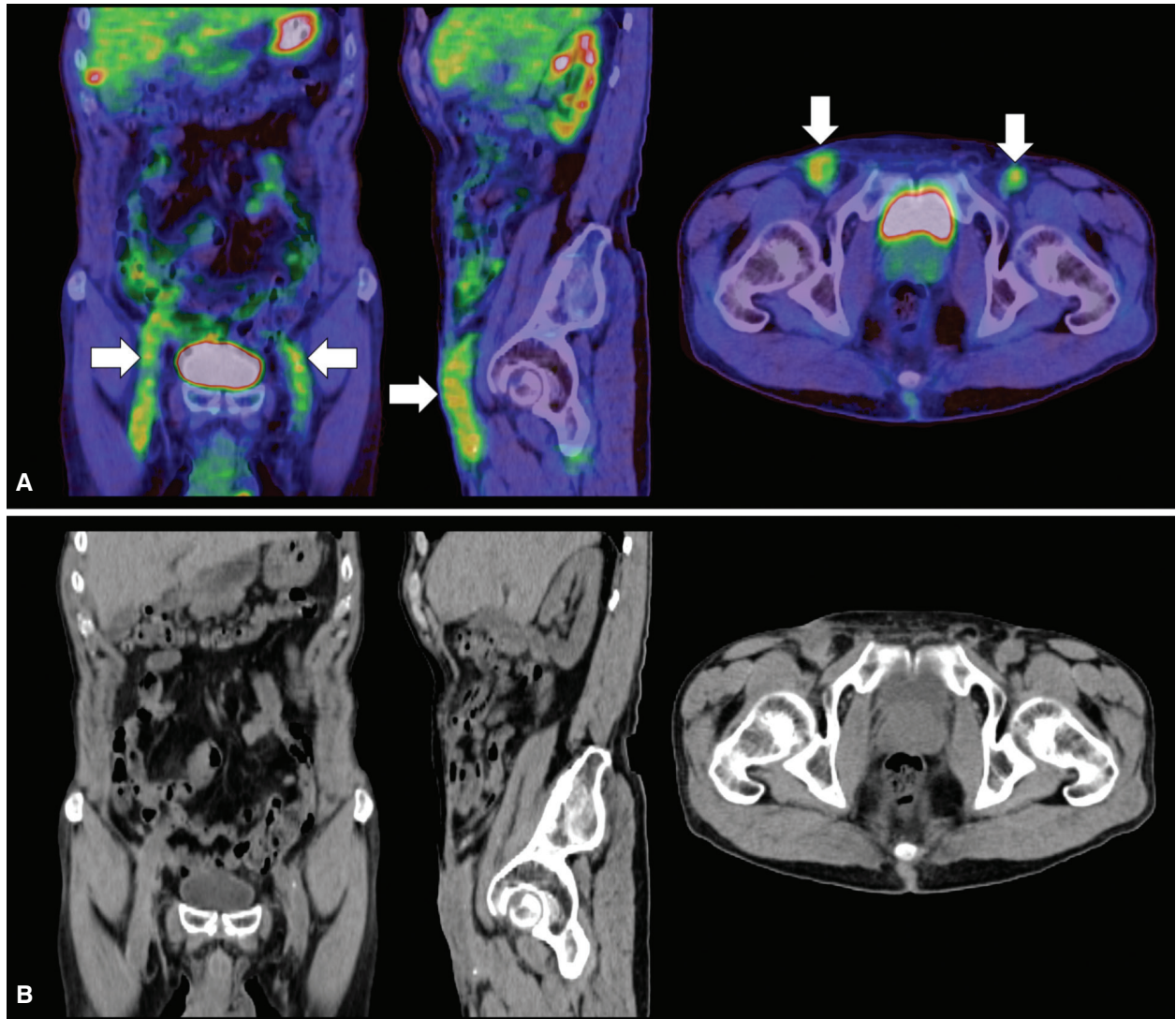

Figure 6. The aorto-femoral prosthesis in a 61-year-old male patient, performed 6 months after replacement of silver salts inlaid prosthesis and implantation of biological prosthesis. No general signs or symptoms of infection were noted in this patients. A. 18F-FDG PET/CT showed quite high metabolic rate in prosthesis bilaterally (arrows); B. CT adapted from 18F-FDG PET/CT — inflammation reaction post biological graft implantation
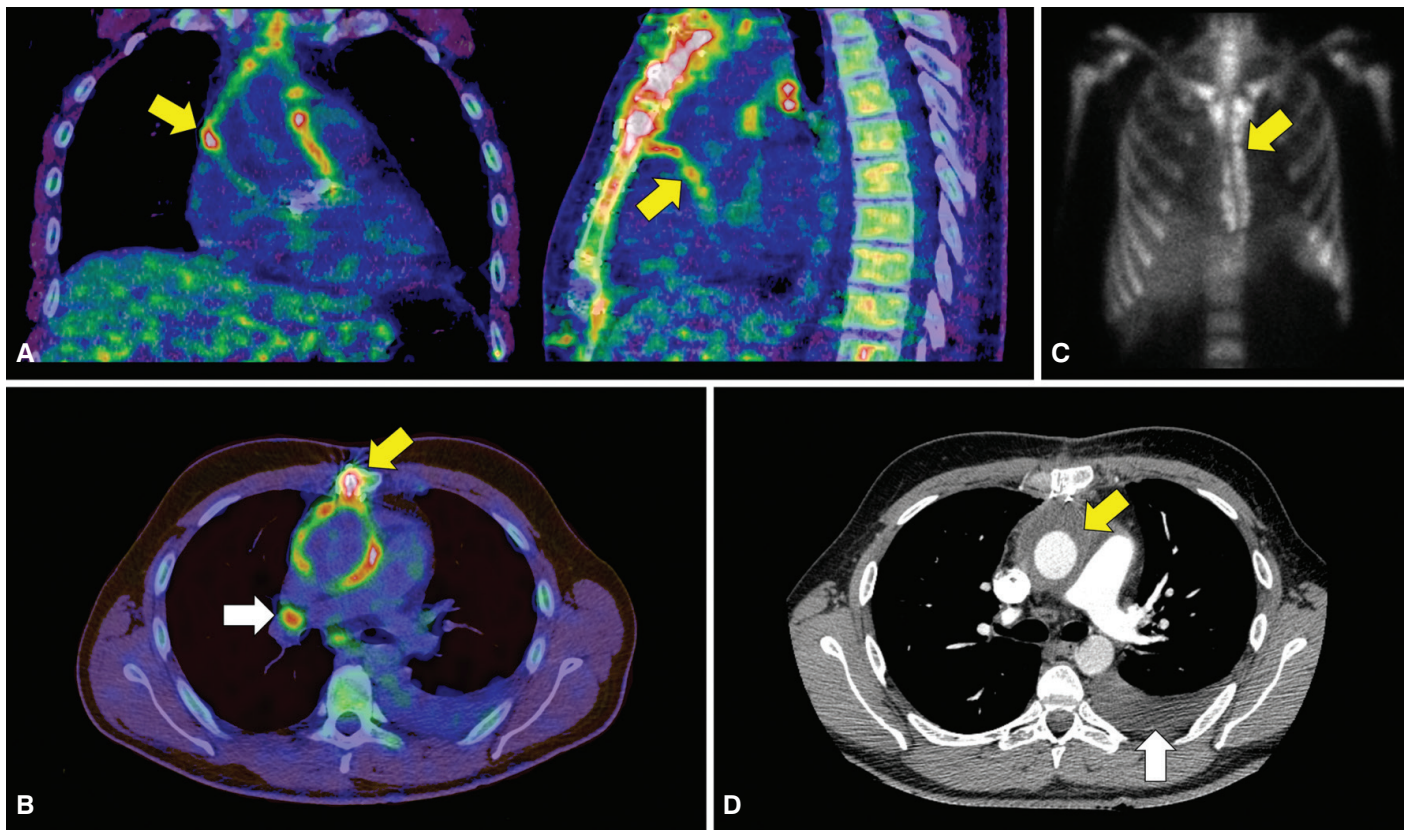

Figure 7. A 36-year-old male patient, one month after Bentall procedure with sternotomy, with persistent fever and elevated biochemical markers of inflammation. A. High uptake of 18F-FDG in PET/CT coronal and sagittal slices (yellow arrows) in the mediastinum around the prosthesis of ascending aorta, without obvious uptake of FDG in prosthesis and in the aortic valve; B. High metabolic activity in sternum (white row) and in the lymph nodes (red arrow); C. Granulocyte antibody scintigraphy reveals lack of activity in bone marrow of the sternum (yellow arrow). Despite early post-surgery period, this feature, together with high uptake of 18F-FGD in that bone, suggests osteomyelitis and infections by continuance in the mediastinum; D. Contrast enhanced CT visualized dense fluid around the graft (yellow arrow), effusion in the left pleural cavity (white arrow) 


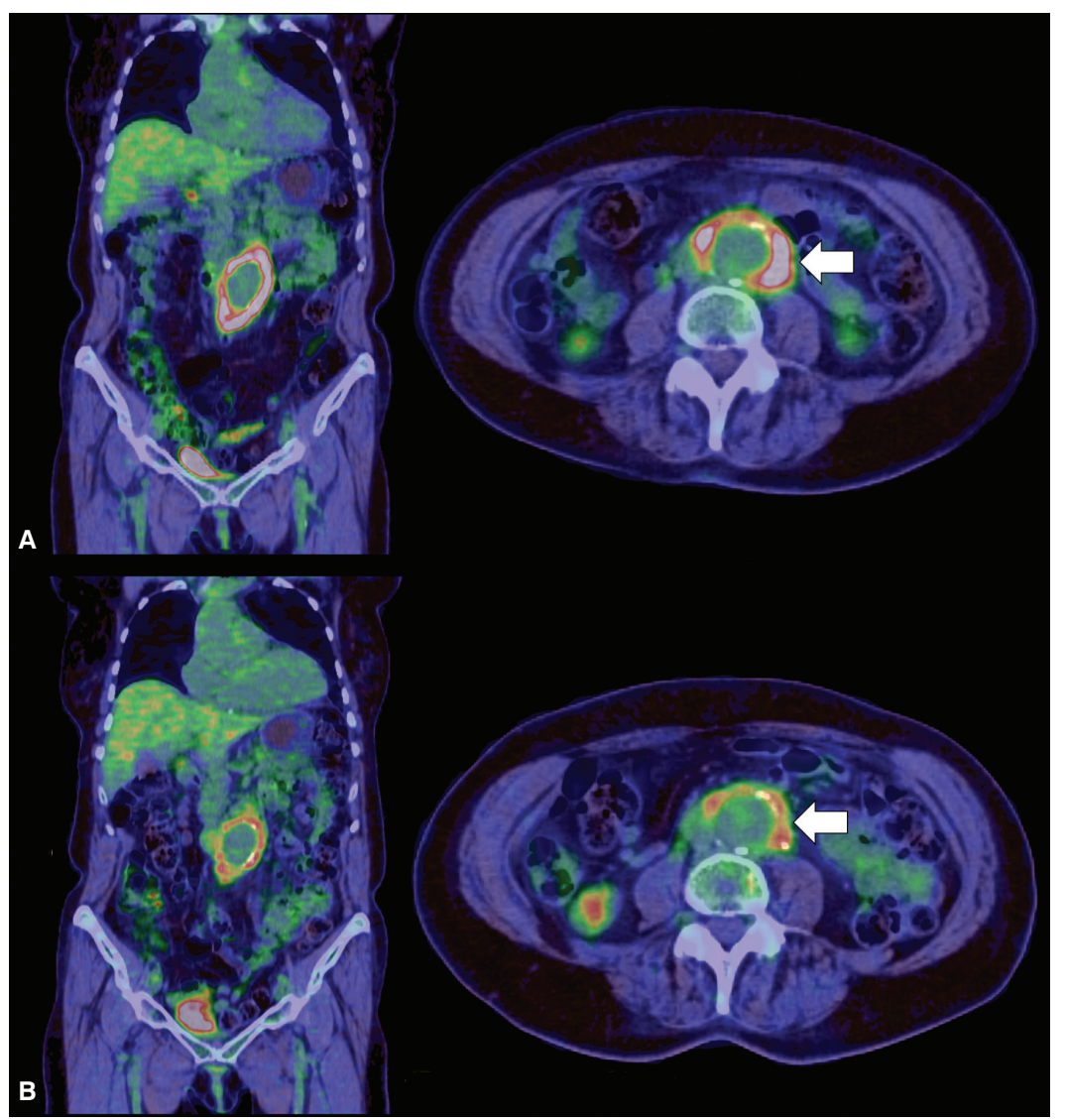

Figure 8. Chronic periaortitis in a 65-year-old female patient with recurrent urinary tract infections and periodontal infections. A. FDG PET/CT before therapy. High uptake of FDG in the tissue around the aneurysm (SUV max 8); B. FDG PET/CT only after reducing sources of infections (tooth extraction, DJ renal catheter). Decreased uptake of FDG around the aneurysm (SUV max 5)

a five-point scale, where grade 0 corresponds to FDG uptake in prosthesis similar to the background, grade 1 - FDG uptake in muscles and fat, grade 2 is defined as clearly visible, grade 3 - strong FDG uptake, but lower than physiologic uptake in bladder, grade 4 - very strong - comparable with bladder. Grades 3 and 4 were classified as infection. High sensitivity of 91-93\% and specificity of $91-95 \%[45,46]$, as well as high positive predictive value of $88 \%$ and negative predictive value of $96 \%$ were established for this method [46]. Bruggink et al. proved that single FDG-PET had better sensitivity $93 \%$, specificity $70 \%$, positive predictive value $82 \%$ and negative predictive value $88 \%$ compared with CT: $56 \%$, $57 \%, 60 \%$ and $58 \%$, respectively [48]. The authors also underline the influence of antibiotics therapy on detection of $\mathrm{VGl}$, which is often very aggressive and may change well-detectable active infections in low-grade process and therefore produces false negative results, especially in single CT study. Vascular graft replacement is associated with higher false positive results [44].

\section{Chronic periaortitis}

Chronic periaortitis (CP) is also known as retroperitoneal fibrosis or Ormond disease. It is an excessive fibro-inflammatory reaction, that starts in the adventitia of abdominal aorta and iliac arteries and extends into the retroperitoneum and also often leads to the involvement of adjacent structures (ureters, inferior vena cava). It is characterized by the presence of retroperitoneal and paraspinal fibrous mass [5]. This infiltration consists of fibroblasts, lympho- cytes, macrophages and vascular endothelial cells [5]. Retroperitoneal fibrosis is commonly idiopathic, but also is associated with certain drugs, systemic disorders and malignant diseases. Chronic periaortitis includes three main entities: idiopathic retroperitoneal fibrosis (IRF), inflammatory abdominal aortic aneurysms (IAAAs), and perianeurysmal retroperitoneal fibrosis (PRF) [49-52]. Figure 8 presents PRF in female patient with recurrent urinary tract infections and periodontal infections, which was presumed as a triggering factor of the disease.

The signs of inflammation with increased levels of inflammatory markers are present in the clinical picture. CP is often linked with immunologic disease, such as systemic vasculitis [53]. Idiopathic retroperitoneal fibrosis is considered as an excessive inflammatory response to atheromatic plaque antigens, such as oxidized low-density lipoproteins and ceroid [50, 52, 54]. Salvarani et al. [52] applied 18F-FGD PET/CT for detection of presence and extent of a large-vessel inflammation in patients with CP. The authors observed seven patients with CP during 3 years. The increased accumulation of FDG indicated the inflammatory process, mainly localized in the abdominal aorta and/or in the common iliac arteries. In $43 \%$ of the patients thoracic aorta and branches were also involved. FDG findings were similar to GCA and TA. According to the authors, the data suggest that $\mathrm{CP}$ may represent a large-vessel vasculitis involving the abdominal aorta and common iliac arteries, which in some patients also extends to the thoracic aorta and/or its branches [52]. 


\section{Conclusions}

In our review we stress the feasibility and value of 18F-FDG PET in vascular inflammation/infection depiction. The whole-body 18F-FDG PET/CT imaging is an important, non-invasive, reliable diagnostic tool used in detecting and monitoring of infections and inflammations in vascular system. This method allows for immediate assessment of response of anti-inflammatory treatment and guides a therapy. It is obvious that contemporary imaging of infections or inflammation, especially using FDG requires involving hybrid modalities.

We do believe that in each case of problematic diagnosis of vascular infection or inflammation FDG imaging of activated leucocytes brings an added value. For proper diagnosis of vascular infections, a diagnostic team should include a nuclear medicine physician, a radiologist and a vascular surgeon. Obviously, the final decision depends on the results of bacteriological and histopathological examinations.

\section{References}

1. Jamar F, Buscombe J, Chiti A et al. EANM/SNMMI guideline for 18F-FDG use in inflammation and infection. Journal of Nuclear Medicine 2013; 54: 647-658.

2. Signore A, Glaudemans AWJM. The molecular imaging approach to image infections and inflammation by nuclear medicine techniques. Annals of Nuclear Medicine 2011; 25: 681-700.

3. Ben-Haim S, Gacinovic S, Israel O. Cardiovascular infection and inflammation. Semin Nucl Med 2009; 39: 103-114.

4. Jennete JC, Falk RJ, Andassy K et al. Nomenclature of systemic vasculitides. Proposal of an internatonal consensus conference. Arthritis Rheum 1994; 37: 187-192

5. Restrepo CS, Ocazionez D, Suri R, Vargas D. Aortitis: imaging spectrum of the infectious and inflammatory conditions of the aorta. RadioGraphics 2011; 31: 435-451.

6. Jennete JC, Falk RJ, Bacon PA et al. Revised International Chapel Hill Consensus Conference Nomenclature of Vasculitides. Arthritis \& Rheumatism 2013; 65: 1-11.

7. Quinn KT Ng, Klaeser B, Walker UA, Walter MA. FDG-PET in large vessel vasculitis, advances in the diagnosis and treatment of vasculitis. Amezcua-Guerra LM (Ed.) 2011.

8. Lande A. Abdominal Takayasu's aortitis, the middle aortic syndrome and atherosclerosis. A critical review. Int Angiol 1998; 17: 1-9.

9. Tezuka D, Haraguchi G, Ishihara T et al. Role of FDG PET-CT in Takayasu Arteritis. Sensitive Detection of Recurrences. J Am Coll Cardiol Img 2012; 5: 422-429.

10. Kobayashi $\mathrm{Y}$, Ishii K, Oda K et al. Aortic wall inflammation due to Takayasu arteritis imaged with 18F-FDG PET coregistered with enhanced CT. J Nucl Med 2005; 46: 917-922.

11. Restrepo CS, Ocazionez D, Suri R, Vargas D. Aortitis: Imaging Spectrum of the Infectious and Inflammatory Conditions of the Aorta. RadioGraphics 2011; 31: 435-451

12. Desai MY, Stone JH, Foo TK, Hellmann DB, Lima JA, Bluemke DA. Delayed contrast-enhanced MRI of the aortic wall in Takayasu's arteritis: initial experience. AJR Am J Roentgenol 2005; 184: 1427-1431.

13. Gotway MB, Araoz PA, Macedo TA et al. Imaging findings in Takayasu's arteritis. AJR Am J Roentgenol 2005; 184: 1945-1950.

14. Nastri MV, Baptista LP, Baroni RH et al. Gadolinium-enhanced three-dimensional MR angiography of Takayasu arteritis. RadioGraphics 2004; 24: 773-786

15. Salvarani C, Gabriel SE, O'Fallon WM, Hunder GG. The incidence of giant cell arteritis in Olmsted County, Minnesota: apparent fluctuations in a cyclic pattern. Ann Intern Med 1995; 123: 192-194.
16. Salvarani C, Macchioni PL, Tartoni PL et al. Polymyalgia rheumatica and giant cell arteritis: a 5-year epidemiologic and clinical study in Reggio Emilia, Italy. Clin Exp Rheumatol 1987; 5: 205-215.

17. Bley TA, Ghanem NA, Foell D et al. Computed tomography coronary angiography with 370-millisecond gantry rotation time: evaluation of the best image reconstruction interval. J Comput Assist Tomogr 2005; 29: 1-5.

18. Pacini D, Leone O, Turci S et al. Incidence, etiology, histologic findings, and course of thoracic inflammatory aortopathies. Ann Thorac Surg 2008; 86: 1518-1523.

19. Vanderschueren S, Knockaert D, Adriaenssens $T$ et al. From prolonged febrile illness to fever of unknown origin. The challenge continues. Arch Intern Med 2003; 163: 1033-1041.

20. Martínez-Rodríguez I, Martínez-Amador N, Banzo I et al. Assessment of aortitis by semiquantitative analysis of 180-min 18F-FDG PET/CT acquisition images. Eur J Nucl Med Mol Imaging 2014; 41: 2319-2324.

21. Blockmans D, Stroobants S, Maes A, Mortelmans L. Positron emission tomography in giant cell arteritis and polymyalgia rheumatica: evidence for inflammation of the aortic arch. Am J Med 2000; 108: 246-249.

22. Brodmann M, Lipp RW, Passath A, Seinost G, Pabst E, Pilger E. The role of 2-18F-fluoro-2-deoxy-D-glucose positron emission tomography in the diagnosis of giant cell arteritis of the temporal arteries. Rheumatology 2004; 43: 241-242.

23. Bural GG, Torigian DA, Chamroonrat $W$ et al. FDG-PET is an effective imaging modality to detect and quantify age-related atherosclerosis in large arteries. Eur J Nucl Med Mol Imaging. 2008; 35: 562-569.

24. Johnston KW, Rutherford RB, Tilson MD, Shah DM, Hollier L, Stanley JC. Suggested standards for reporting on arterial aneurysms. Subcommittee on Reporting Standards for Arterial Aneurysms, Ad Hoc Committee on Reporting Standards, Society for Vascular Surgery and North American Chapter, International Society for Cardiovascular Surgery. J Vasc Surg 1991; 13: 452-458.

25. U.S. Preventive Services Task Force. Screening for Abdominal Aortic Aneurysm: Recommendation Statement. Ann Intern Med 2005; 142: 198-202.

26. Huber TS, Wang JG, Derrow AE. Experience in the United States with intact abdominal aortic aneurysm repair. J Vasc Surg 2001; 33: 304-310.

27. Nevitt MP, Ballard DJ, Hallett JW Jr. Prognosis of abdominal aortic aneurysms population-based study. N Engl J Med 1989; 321: 1009-1014.

28. Glimåker H, Holmberg L, Elvin A et al. Natural history of patients with abdominal aortic aneurysm. Eur J Vasc Surg 1991; 5: 125-130.

29. Lederle FA, Johnson GR, Wilson SE et al. Rupture rate of large abdominal aortic aneurysms in patients refusing or unfit for elective repair. JAMA 2002; 287: 2968-2972.

30. Powell JT, Gotensparre SM, Sweeting MJ, Brown LC, Fowkes FG, Thompson SG. Rupture rates of small abdominal aortic aneurysms: a systematic review of the literature. Eur J Vasc Endovasc Surg 2011; 41: 2-10.

31. Georgakarakos E, loannou CV. Geometrical factors as predictors of increased growth rate or increased rupture risk in small aortic aneurysms. Med Hypotheses 2012; 79: 71-73.

32. Courtois A, Nusgens BV, Hustinx R et al. 18F-FDG Uptake Assessed by $\mathrm{PET} / \mathrm{CT}$ in Abdominal Aortic Aneurysms Is Associated with Cellular and Molecular Alterations Prefacing Wall Deterioration and Rupture. J Nucl Med 2013; 54: 1740-1747.

33. Michel JB, Thaunat O, Houard X, Meilhac O, Caligiuri G, Nicoletti A. Topological determinants and consequences of adventitial responses to arterial wall injury. Arterioscler Thromb Vasc Biol. 2007; 27: 1259-1268.

34. Sakalihasan N, Limet R, Defawe OD. Abdominal aortic aneurysm. Lancet 2005; 365: 1577-1589.

35. Liapis $\mathrm{CD}$, Paraskevas KI. The pivotal role of matrix metalloproteinases in the development of human abdominal aortic aneurysms. Vasc Med 2003; 8: 267-271.

36. Reeps C, Essler M, Pelisek J, Seidl S, Eckstein H-H, Krause B-J. Increased 18F-fluorodeoxyglucose uptake in abdominal aortic aneurysms in positron emission/computed tomography is associated with inflammation, aortic wall instability, and acute symptoms. J Vasc Surg 2008; 48: 417-424. 
37. Barwick TD, Lyons OT, Mikhaeel NG, Waltham M, O'Doherty MJ. 18F-FDG PET-CT uptake is a feature of both normal diameter and aneurysmal aortic wall and is not related to aneurysm size. Eur J Nucl Med Mol Imaging 2014; 41: 2310-2318.

38. Cavalcanti Filho JLG, de Souza Leão Lima R, de SouzaMachado Neto L, Kayat Bittencourt L, Cortes Domingues, Barbosa da Fonseca LM. PET/CT and vascular disease: current concepts. European Journal of Radiology 2011; 80: 60-67.

39. Setacci C, Müller-Hülsbeck S, Jamar FX. Common diagnostic flowcharts in vascular and endovascular surgery. Q J Nucl Med Mol Imaging 2014; 58: 46-54.

40. Hasse B, Husmann L, Zinkernager A et al. Vascular graft infections; Swiss Med Wkly. 2013; 143: 13754.

41. Legout L, D'Elia PV, Sarraz-Bournet B. Diagnosis and management of prosthetic vascular graft infections. Med Mal Infect 2012; 42: 102-109.

42. Fiorani P, Speziale F, Rizzo L et al. Detection of aortic graft infection with leukocytes labeled with technetium 99m-hexametazime. J Vasc Surg 1993; 17: 87-95

43. Tokuda Y, Oshima H, Araki Y, Narita Y, Mutsuga M, Kato K, Usui A. Detection of thoracic aortic prosthetic graft infection with 18F-fluorodeoxyglucose positron emission tomography/computed tomography. Eur J Cardiothorac Surg 2013; 43: 1183-1187.

44. van der Vaart MG, Meerwaldt R, Slart RHJA, van Dam GM, Tio RA, Zeebregts CJ Application of PET/SPECT imaging in vascular disease. Eur J Vasc Endovasc Surg 2008; 35: 507-513.
45. Fukuchi K, Ishida $\mathrm{Y}$, Higashi $\mathrm{M}$ et al. Detection of aortic graft infection by Fluorodeoxyglucose positron emission tomography: comparison with computed tomographic findings. J Vasc Surg 2005; 42: 919-925.

46. Keidar Z, Engel A, Hoffman A, Israel O, Nitecki S. Prosthetic Vascular Graft Infection: The Role of 18F-FDG PET/CT. J Nucl Med 2007; 48: 1230-1236.

47. Wasselius J, Malmstedt J, Kalin B et al. High 18F-FDG Uptake in Synthetic Aortic Vascular Grafts on PET/CT in Symptomatic and Asymptomatic Patients. J Nucl Med 2008; 49: 1601-1605.

48. Bruggink JL, Glaudemans AW, Saleem BR et al. Accuracy of FDG-PET-CT in the diagnostic work-up of vascular prosthetic graft infection. Eur J Vasc Endovasc Surg 2010; 40: 348-354.

49. Mitchinson MJ. Chronic periaortitis and periarteritis. Histopathology 1984; 8: 589-600.

50. Parums DV. The spectrum of chronic periaortitis. Histopathology 1990; 16 : 423-431.

51. Vaglio A, Buzio C. Chronic periaortitis: a spectrum of diseases. Curr Opin Rheumatol 2005; 17: 34-40.

52. Salvarani C, Pipitone N, Versari A et al. Positron Emission Tomography (PET): Evaluation of Chronic Periaortitis Arthritis \& Rheumatism (Arthritis Care \& Research) 2005; 53: 298-303.

53. Haug ES, Skomsvoll JF, Jacobsen G, Halvorsen TB, Saether OD, Myhre $\mathrm{HO}$. Inflammatory aortic aneurysm is associated with increased incidence of autoimmune disease. J Vasc Surg 2003; 38: 492-497.

54. Parums DV, Chadwick DR, Mitchinson MJ. The localisation of immunoglobulin in chronic periaortitis. Atherosclerosis 1986; 61: 117-123. 